\title{
NON-LINEAR EFFECTS IN FLAT GRAVITATING SYSTEMS
}

A. M. Fridman

It is shown that in flat gravitating systems, such as spiral galaxies, groups of galaxies and some superclusters (which have an axis ratio of, say, 1/5) there are nonlinear effects such as the formation of collapsars in disks which are stable according to Toomre's criterion; the formation of solitons (spiral and ring-shaped), which can move with supersonic as well as subsonic velocities depending on the characteristic parameters of the disk. In an isothermal gas, shock waves can only originate in spirals with two or more arms. In the background of two bright spiral arms one may be able to trace a weaker ring structure. In contrast to supersonic spirals, the velocity of the circular structure is subsonic. A bright ring structure can in principle indicate that in such systems for some reason the adiabatic index $\gamma$ is close to that of an ideal gas $\gamma \approx 5 / 3$. This last point can be of interest in the future if non-isothermal perturbations will be found as seems possible in some systems. A break-up instability will lead to practically uniform enhanced star formation all over the disk; it will have a tendency to simulate narrow rings and tightly wound spirals with one arm according to the law $\left(\delta \sigma / \sigma_{0}\right) \sim\left(t-t_{0}\right)^{-1}$.

The condition for applicability of the model of interacting galaxies is $(M / m) \geqslant 2 R / h$, where $R, H, m$ are radius, thickness and mass of the disk and $M$ is the mass of the galaxy centre (the bulge component). For spiral galaxies, $(\mathrm{M} / \mathrm{m})_{\max } \simeq 2,2 \mathrm{R} / \mathrm{h} \simeq 20$ to 30 , therefore condition of applicability of the model of interacting galaxies is not fulfilled. 